\section{Commercially Available Insulin Products Demonstrate Stability Throughout the Cold Supply Chain Across the U.S.}

Diabetes Care 2020;43:1360-1362 | https://doi.org/10.2337/dc19-1941

\section{OBJECTIVE}

A recent publication questioned the integrity of insulin purchased from U.S. retail pharmacies. We sought to independently validate the method used, isotope dilution solid-phase extraction (SPE) liquid chromatography mass spectrometry (LC-MS), and expand analysis to two U.S. Pharmacopeia (USP) methods (highperformance LC with ultraviolet detection and LC-MS).

\section{RESEARCH DESIGN AND METHODS}

Each method was used to evaluate nine insulin formulations, purchased at four pharmacies, within five geographic locations in the U.S.

\section{RESULTS}

All human and analog insulins measured by the USP methods $(n=174)$ contained the expected quantity of active insulin (100 \pm 5 units $/ \mathrm{mL})$. When using isotope dilution SPE-LC-MS, units-per-milliliter values were well below product labeling due to unequal recovery of the internal standard compared with target insulin.

\section{CONCLUSIONS}

Insulin purchased from U.S. pharmacies is consistent with product labeling.

Insulin formulations are subjected to rigorous testing prior to their release from pharmaceutical manufacturers, as required by the U.S. Food and Drug Administration and the European Medicines Agency (1). However, a recent study by Carter and Heinemann (2) examined vials of regular and intermediate-acting NPH insulin purchased from local pharmacies in the U.S., and remarkably, almost none met the required standard of 100 units $/ \mathrm{mL}$ ( \pm 5 units $/ \mathrm{mL}$ ) set forward by the U.S. Pharmacopeia (USP). Insulin stability is of critical importance for both the safety and efficacy of diabetes treatment (3-5). Hence, the Carter and Heinemann (2) study, which did not use a USP-approved method, was widely recognized by both national and social media, raising concerns among care providers and patients whose confidence was disrupted regarding the potential reliability of insulin to reproducibly manage their disease $(6,7)$. Therefore, we devised a well-powered and independent assessment of insulin products (e.g., vials vs. pens and human vs. analog) from major manufacturers using approved USP methods and comparable liquid chromatography mass spectrometry (LC-MS) methods $(8,9)$, along with the method of the aforementioned report (2), to provide an assessment of the insulin concentration purchased at retail pharmacies.
Timothy J. Garrett, ${ }^{1}$ Philip Atkinson, ${ }^{2}$ Eoin P. Quinlivan, ${ }^{1}$ Lynn Ang, ${ }^{3}$ Irl B. Hirsch, ${ }^{4}$ Lori Laffel, ${ }^{5}$ Massimo Pietropaolo, ${ }^{6}$ Michael J. Haller, ${ }^{7}$ and Mark A. Atkinson ${ }^{1,2,7}$
${ }^{1}$ Department of Pathology, Immunology and Laboratory Medicine, College of Medicine, University of Florida, Gainesville, FL ${ }^{2}$ Insulin for Life USA, Gainesville, FL

${ }^{3}$ Division of Metabolism, Endocrinology \& Diabetes, Department of Internal Medicine, University of Michigan, Ann Arbor, MI

${ }^{4}$ Endocrine Care Center, University of Washington Medical Center-Roosevelt, Seattle, WA ${ }_{5}^{5}$ Joslin Diabetes Center, Harvard Medical School, Boston, MA

${ }^{6}$ Department of Pathology and Immunology, Baylor College of Medicine, Houston, TX

${ }^{7}$ Department of Pediatrics, College of Medicine, University of Florida Diabetes Institute, Gainesville, $F L$

Corresponding author:TimothyJ.Garrett, tgarrett@ ufl.edu

Received 30 September 2019 and accepted 20 March 2020

This article contains Supplementary Data online at https://doi.org/10.2337/dc20-1234/ suppl.12016422.

(C) 2020 by the American Diabetes Association. Readers may use this article as long as the work is properly cited, the use is educational and not for profit, and the work is not altered. More information is available at https://www.diabetesjournals .org/content/license. 


\section{RESEARCH DESIGN AND METHODS}

\section{Study Design}

Insulin activity was quantitatively assessed from rapid-, short-, intermediate-, longacting, and premixed formulations produced by three major manufacturers (i.e., Eli Lilly and Company, Novo Nordisk, and Sanofi). Nine insulin products (Supplementary Table 1) were acquired from four pharmacy or grocery-chain retail providers within five U.S. geographical regions (Seattle, WA [Northwest]; Houston, TX [Southwest]; Gainesville, FL [Southeast]; Boston, MA [Northeast]; and Ann Arbor, MI [Midwest]) for a total of $\sim 20$ replicates/product and 174 total samples. Immediately following purchase, the insulins were placed into thermostable coolers with temperature-recording devices and transported to the University of Florida for analysis. No disruptions in insulin temperature outside the acceptable range were identified.

\section{Insulin Analysis}

For high-performance LC with ultraviolet detection (LC-UV) analysis, insulin samples from each site/region were prepared for detection as described in their respective official USP Monographs (Supplementary Table 2). Chromatographic separation and detection were achieved using a Vanquish UHPLC Variable Wavelength Detector and the data analyzed using Chromeleon 7. For LC-MS analysis, insulin standards and samples were analyzed using a Thermo $Q$ Exactive Orbitrap mass spectrometer with Dionex UHPLC. Additional procedures are provided in the Supplementary Materials and Methods. Finally, we replicated the non-USP methodology (2) (Supplementary Materials and Methods).

\section{Statistics}

The variables for statistical analysis were as follows: 1) insulin product, 2) pharmacy, 3) region, and 4) units per milliliter. Quantitative results from each method were compared against the same method and among different methods used to assess the quality of insulin measurement and insulin activity in each formulation. Accuracy was defined as how similar the measured value was to the actual value and calculated based on the labeled concentration of 100 units $/ \mathrm{mL}$, while precision was calculated based on the mean of the replicates divided by the SD, represented as relative SD.
Data and Resource Availability

The data sets generated and analyzed during the current study are available from the corresponding author upon reasonable request.

\section{RESULTS}

Using the analytical criteria described in USP Monographs to test the concentration of our insulin samples, all five human insulins were noted within the acceptable range of $100 \pm 5$ units/mL (Fig. 1 and Supplementary Table 3). In addition, these efforts confirmed that the standard USP LC-UV method was highly reproducible.

Given the disparate insulin concentrations observed between our analysis using USP LC-UV methods and those reported for solid-phase extraction (SPE)LC-MS (2), we replicated that assay, an effort that consistently noted lower unitsper-milliliter values compared with the USP LC-UV method for all five human insulins (Fig. 1) $(P<0.0001)$. No degradation products were observed in the spectra to account for the difference reported. However, we noted a large disparity in recoveries between the hu$\operatorname{man}(20.6 \pm 1.6 \%)$ and bovine (129.5 \pm $1.4 \%$ ) insulin (internal standard) from standard solutions (10). For extracted samples, recovery of human insulin was slightly lower (18.1 $\pm 2.3 \%)$ and bovine insulin slightly higher (144.8 $\pm 12.8 \%$ ). This difference in recovery between standards and samples was sufficient to cause a $25 \%$ decrease in calculated human insulin concentrations. We then assessed the recovery of bovine insulin in relation to human insulin through comparison with MS analysis without SPE. The results indicated a differential extraction efficiency, which would cause the quantitative errors observed. Specifically, using a milder aqueous diluent in the extraction process enabled similar recovery for the internal standard and measured insulin, helping to account for any insulin loss during the extraction process (Supplementary Table 4).

Finally, we assessed the stability of insulin analogs using only two methods (USP LC-UV and LC-MS). The SPE-LC-MS method was not used for the analogs given the results obtained for human insulin. All analogs measured by the USP methods reported acceptable mean values for insulin analog activity with the mean of the replicates divided by the SD, represented as relative SD, typically $<1 \%$ (Supplementary Table 3). While there was a statistically significant difference in some regional samples (e.g., NovoLog from the Southeast compared with NovoLog from the Northwest), these variations were not considered clinically significant as the values were all within the acceptable range of $100 \pm$ 5 units/mL.

\section{CONCLUSIONS}

The effort reported in this study represents an important independent analysis seeking to identify potential failures in
USP values of Human Insulin reported by 3 methods

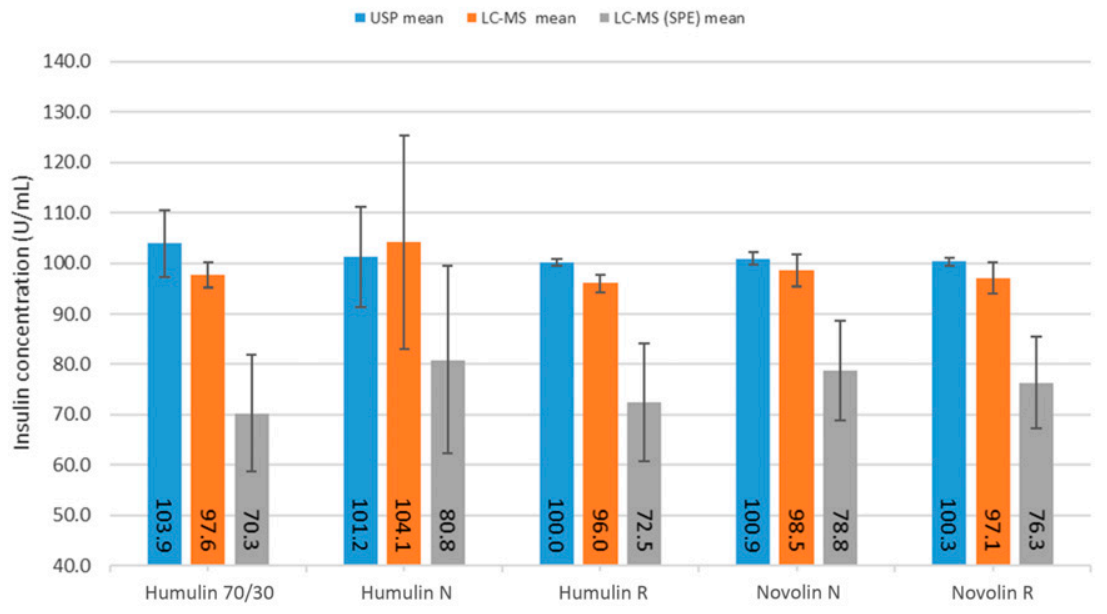

Figure 1-Human insulin products reported by three analysis methods. Human insulins were measured by USP LC-UV (blue), LC-MS without SPE (orange), and SPE-LC-MS (gray) (2) and reported as USP insulin units per milliliter, mean $\pm 1 \mathrm{SD}$. 
the cold supply chain emanating from geographic region and/or pharmacy that could affect the quality and reliability of insulin products at the point-of-patient purchase across the U.S. The study also provides a comprehensive assessment of insulin analysis methods to ensure reliability of measurement. Analysis of insulins from five geographic locations within the U.S. provided a panoramic overview of multiple possible variables by proxy, including potential impacts of shipping duration, weather/temperature during insulin distribution, as well as local/regional variation resulting from warehouse/wholesale distributors, potentially in rural and urban areas.

We believe our study, thus far, provides an affirming answer to the question of the viability of the U.S. insulin supply at the point of retail pharmacies. In this study, we report the data collected from insulins purchased in the winter of 2018 to 2019, representing the first analysis of an ongoing effort involving a 1-year, 2-phase study in which we will analyze activity from 720 pharmaceutical insulin samples purchased over 4 seasons. These data in this study (phase 1) will be expanded (phase 2) to measure potential seasonal variations in reported insulin activity Should we identify deficits in insulin stability from one or more regions during phase 2 of the study, it will be important to identify specific factors impeding the successful delivery of viable insulin.

Other recent research efforts, albeit smaller in scope or variant in their study design, have also addressed this concern. One report by Moses et al. (11) noted samples from Novo Nordisk were delivered within USP requirements, while a second study using separate methodology also confirms consistency with USP requirements (12). These reports further add to the notion of insulin safety.

We acknowledge that other issues may further influence quality or consistency of insulin that reside outside of our current investigations. These include, but are not limited to: 1 ) humidity, 2) insulin concentrations (e.g., U40, U100, U300, and U500), 3) mail-order versus direct (i.e., local pharmacy) purchase, 4) disparities related to rural versus urban areas and socioeconomic landscape, and 5) international issues related to cold chain supply outside the U.S. We recognize these and would be interested in pursuing such issues moving forward. However, the present data should serve to quell questions regarding the viability of insulin purchased at retail pharmacies in the U.S.

Funding. These studies were supported by JDRF, American Diabetes Association, and The Leona M. and Harry B. Helmsley Charitable Trust.

The funders were consulted regarding study design to determine the number of insulin products and pharmacies for analysis. However, the funding sources were not involved in the collection, analysis, and interpretation of the data, nor did they influence the writing of the report or the decision to submit the manuscript for publication.

Duality of Interest. No potential conflicts of interest relevant to this article were reported. Author Contributions. T.J.G. researched the data and wrote the manuscript. P.A. coordinated the study, contributed to the discussion, and reviewed and edited the manuscript. E.P.Q. performed the sample analysis, performed initial data analysis, contributed to the discussion, and reviewed and edited the manuscript. L.A., I.B.H., L.L., M.P., and M.J.H. served as the study site principal investigators, contributed to discussion, and reviewed and edited the manuscript. M.A.A. conceived of the study and wrote the manuscript. T.J.G. is the guarantor of this work and, as such, had full access to all of the data in the study and takes responsibility for the integrity of the data and the accuracy of the data analysis.

\section{References}

1. Koch W, Ma B. The US Pharmacopeia: interfacing chemical metrology with pharmaceutical and compendial science. Accred Qual Assur 2011;16:43-51

2. Carter AW, Heinemann L. Insulin concentration in vials randomly purchased in pharmacies in the United States: considerable loss in the cold supply chain. J Diabetes Sci Technol 2018;12:839-841

3. Grajower MM, Fraser CG, Holcombe JH, et al. How long should insulin be used once a vial is started? Diabetes Care 2003;26:2665-2666; discussion 266-269

4. Brange J, Langkjœer L. Insulin structure and stability. In Stability and Characterization of Protein and Peptide Drugs: Case Histories. Wang YJ, Pearlman R, Eds. Boston, MA, Springer US, 1993, p. 315-350

5. Jeffrey PD, Milthorpe BK, Nichol LW. Polymerization pattern of insulin at $\mathrm{pH}$ 7.0. Biochemistry 1976;15:4660-4665

6. Petersen MP, Hirsch IB, Skyler JS, Ostlund RE, Cefalu WT. In response to Carter and Heinemann: insulin concentration in vials randomly purchased in pharmacies in the United States: considerable loss in the cold supply chain. J Diabetes Sci Technol 2018;12:890-891

7. Connery A, Martin S. Lilly calls into question the validity of published insulin concentration results. J Diabetes Sci Technol 2018;12:892-893

8. Vanhee C, Janvier S, Moens G, Deconinck E, Courselle P. A simple dilute and shoot methodology for the identification and quantification of illegal insulin. J Pharm Anal 2016;6:326-334

9. Loos G, Van Schepdael A, Cabooter D. Quantitative mass spectrometry methods for pharmaceutical analysis. Philos Trans Royal Soc Math Phys Eng Sci 2016;374:20150366

10. Bronsema KJ, Bischoff $R$, van de Merbel NC. Internal standards in the quantitative determination of protein biopharmaceuticals using liquid chromatography coupled to mass spectrometry. J Chromatogr B Analyt Technol Biomed Life Sci 2012;893-894:1-14

11. Moses A, Bjerrum J, Hach M, Wæhrens LH, Toft AD. Concentrations of intact insulin concurs with FDA and EMA standards when measured by HPLC in different parts of the distribution cold chain. J Diabetes Sci Technol 2019;13:55-59 12. Malmodin D, Pedersen A, Karlsson BG, Forsander G. NMR spectroscopic analysis to evaluate the quality of insulin: concentration, variability, and excipient content. J Diabetes Sci Technol 2020;14:180-184 